\title{
Innovative Methoden in Fließgewässermonitoring, Modellierung und Flussbau
}

\begin{abstract}
Wasser stellt eine Grundvoraussetzung für Leben dar, kann aber bei Hochwasser auch Leben zerstören. Flüsse sind die Lebensadern der Landschaft und die meisten menschlichen Siedlungen befinden sich an diesen, verbunden mit allen Nutzungsmöglichkeiten wie Schifffahrt, Trinkwasser, Energiewirtschaft, aber auch mit Gefahren. Die Nutzung der Flüsse steht zunehmend im Konflikt mit deren ökologischer Bedeutung. Viele Zusammenhänge zwischen dem Transport von Wasser und Feststoffen, der Morphologie von Flüssen, der Ökologie und den Auswirkungen von flussbaulichen Maßnahmen sind noch immer unklar.
\end{abstract}

Wesentliche Inhalte dieses ÖWAW-Heftes stammen aus der ersten Phase der Tätigkeit des an der BOKU gemeinsam mit den Firmenpartnern via donau und UWITEC eingerichteten Christian Doppler Labors für Innovative Methoden in Fließgewässermonitoring, Modellierung und Flussbau (IM_FLUSS; http://cdlabor-imfluss.boku.ac.at), wissenschaftlichen Untersuchungen an der Donau östlich von Wien im Auftrag des BMVIT und via donau sowie verschiedenen Forschungsprojekten im Auftrag des Lebensministeriums, der Länder und aus EU-Projekten.

Die Ziele des Christian Doppler Labors IM_FLUSS liegen in der Verbesserung des Verständnisses ablaufender Prozesse in Flüssen (Modul I Fließgewässermonitoring), der Entwicklung von mathematischen Modellen zur Prozessbeschreibung und Prognose der Auswirkungen von flussbaulichen Maßnahmen (Modul II Fließgewässermodellierung) sowie der Entwicklung von innovativen wasserbaulichen Methoden zur Verbesserung der Schifffahrt, des Hochwasserschutzes und der Ökologie (Modul III Flussbau). 2012 wurde in der Phase 2 des CD-Labors in Erweiterung der bisherigen drei Module noch ein Modul IV Wasserkraft genehmigt, das sich gemeinsam mit Partnerunternehmen aus der Energiewirtschaft mit den Themen Schwall und Hydrokinetik beschäftigt.

Das vorliegende Heft beinhaltet zwei Artikel zum Thema Monitoring, zwei Beiträge zur numerischen Modellierung, einen Artikel zu Maßnahmen im modernen Flussbau und einen zur physikalischen Modellierung.

Die Beiträge zum Fließgewässermonitoring (Liedermann et al., Haimann et al.) befassen sich mit Messmethoden und -konzepten zu den Themen Geschiebe- und Schwebstofftransport unter Einsatz innovativer Messgeräte wie Acoustic Doppler Current Profiler (ADCP) und Radiotracer. Dabei geht es um eine Verbesserung des Prozessverständnisses, die Schaffung einer Datenbasis für die Kalibrierung und Validierung mathematischer Modelle und die Optimierung des Monitorings für den Einsatz in der Praxis.

Im Bereich der Fließgewässermodellierung behandeln zwei Artikel (Tritthart et al., Hauer et al.) mehrdimensionale mathematische Modelle zur Simulation der Hydrodynamik, des Sedimenttransportes, der Flussmorphologie sowie der ökologischen Prozesse in Form der Habitatmodellierung. Dabei werden

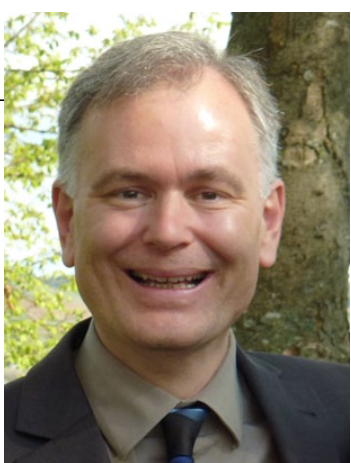

Univ.-Prof. DI

Dr. Helmut Habersack 
die Entwicklung, die innovativen Ansätze, die softwaretechnische Umsetzung und die Anwendung am Beispiel der Donau östlich von Wien dargestellt.

Zum Thema Flussbau diskutieren Habersack et al. die Entwicklung und Umsetzung von innovativen wasserbaulichen Maßnahmen betreffend Sohlstabilisierung und Flussrückbau, insbesondere die Granulometrische Sohlverbesserung, Buhnenoptimierung, Uferrückbau und Gewässervernetzung. Diese dienen beispielsweise sowohl der wirtschaftlichen Entwicklung entlang der Donau als internationale Wasserstraße, als auch der Ökologie. Die Überprüfung der sohlstabilisierenden Wirkung der Granulometrischen Sohlverbesserung mittels physikalischer Modellversuche wird durch Hengl et al. erläutert.

Dank gebührt besonders via donau und UWITEC als Firmenpartnern des Christian Doppler Labors IM_FLUSS, dem BMVIT, dem Lebensministerium, dem Ministerium für Wirtschaft, Familie und Jugend sowie der Nationalstiftung für Forschung, Technologie und Entwicklung, den Ämtern mehrerer Landesregierungen und der EU für die finanzielle und logistische Unterstützung.

\section{Univ.-Prof. DI Dr. Helmut Habersack}

Universität für Bodenkultur Wien

Department für Wasser - Atmosphäre - Umwelt

Institut für Wasserwirtschaft, Hydrologie und konstruktiven Wasserbau

Christian Doppler Labor für Innovative Methoden in Fließgewässermonitoring,

Modellierung und Flussbau

Muthgasse 107

1190 Wien

Österreich

helmut.habersack@boku.ac.at

\section{IMFuse}

CD Labor für Innovative Methoden in Fließgewässermonitoring,

Modellierung und Flussbau

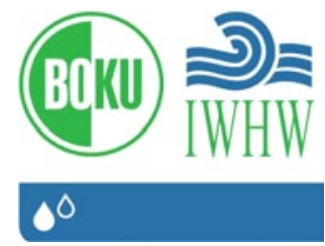

